\title{
Efficacy Report of Lumbar Spinal Stenosis of 90 Cases Based on Adjusting the Curvature of the Spine with Hyperextension Traction
}

\author{
Yizong Wei1, Donghua Pan1, Xiuguang Wang', Chunde Wei', Teng Gao', \\ Shengqiang Zhang ${ }^{2}$, Tingzhang Lin $^{3}$ \\ ${ }^{1}$ Beijing Guangming Orthopedics and Traumatology Hospital, Beijing, China \\ ${ }^{2}$ Foshan Hospital of TCM, Foshan, China \\ ${ }^{3}$ Chaozhou Central Hospital Affiliated to Southern Medical University, Chaozhou, China \\ Email: wftco2002@163.com
}

Received 30 December 2014; accepted 15 January 2015; published 21 January 2015

Copyright (C) 2015 by authors and OALib.

This work is licensed under the Creative Commons Attribution International License (CC BY). http://creativecommons.org/licenses/by/4.0/

\section{(c) (i) Open Access}

\begin{abstract}
Objective: To observe the clinical effect of the treatment of lumbar spinal stenosis disease by adjusting the curvature of the spine based on orthopedic spinal therapy. Method: Curvature adjustments by four-direction, spinal orthopedic manipulation and functional exercise were applied to different patterns of lumbar spinal stenosis. Results: The overall response rate was $98.9 \%$, including a clinical recovery rate of $83.3 \%$, response rate of $13.3 \%$, marked effectiveness rate of $2.2 \%$ and failure rate of $1.1 \%$. The spinal curvatures were improved in $81 \%$ of the patients. The 3 month follow-up showed that the "excellent" and "very good" rates of the health status and signs $\&$ symptoms improvement were $\mathbf{9 8 . 8 \%}$ and $96.4 \%$ respectively. Conclusion: The principal objective of curvature adjustment and Chinese orthopedic manipulation is to adjust the lumbar curvature. Functional exercise is positively correlated with restoration and stability of the spinal curvature. The follow-up indicates that regular exercise is essential to facilitate the therapeutic efficacy.
\end{abstract}

\section{Keywords}

Lumbar Spinal Stenosis, Hyperextension Suspension Traction, Spinal Curvature Adjustment, Spinal Orthopedic Manipulation, Lumbar Curvature

Subject Areas: Clinical Trials, Orthopedics

\section{Introduction}

Lumbar spinal stenosis (LSS) is a medical condition in which the spinal canal narrows and compresses the nerve

How to cite this paper: Wei, Y.Z., Pan, D.H., Wang, X.G., Wei, C.D., Gao, T., Zhang, S.Q. and Lin, T.Z. (2015) Efficacy Report of Lumbar Spinal Stenosis of 90 Cases Based on Adjusting the Curvature of the Spine with Hyperextension Traction. Open Access Library Journal, 2: e1159. http://dx.doi.org/10.4236/oalib.1101159 
of the cauda equina or nerve roots at the level of the lumbar vertebra. This is usually due to the lumbar curvature disorder and then occurrence of spinal disc herniation, disc protruding into the spinal canal, or because of the rotational displacement of vertebral body, posterior longitudinal ligament and ligamentum flavum thickening and fold, and other reasons, resulting in 1 or more intervertebral disc area stenosis.

Between February 2009 and December 2009, we treated a total of 90 LSS cases and obtained satisfactory outcomes with spinal curvature adjustment by hyperextension suspension traction, spinal orthopedic manipulation, acupuncture, oral and external use of Chinese medicine. The results are now reported as follows:

Clinical Materials

General Material

Of the 90 LSS cases, the male/female ratio: 47/43, the age range: 22 - 75 years old, average age: 50; and the duration of disease: 1 - 414 months, average duration: 18 months. Of the 11 rejected cases, 9 failed to stick to the treatment after obvious alleviation of symptoms; 2 cases had slight adverse reactions, which disappeared after treatment and discontinued the treatment.

Diagnostic criteria were based on the "Lumbar Spine" [1] [2].

1) Chronic progressive low back pain was accompanied with leg pain and weakness. Intermittent claudication or claudication needed help;

2) Localized leg dysesthesia or numbness were coupled with tendon reflex decrease and muscle weakness below grade 4; frequent or incontinent urination and weakness in passing stools;

3) Radiographic image showed over $2^{\circ}$ of posterior-anterior (PA) rotation, over $5^{\circ}$ of scoliosis, a smaller, disappeared or even reversed spinal curvature from the lateral view that could be classified as grade III, IV and V;

4) SEP femoral, tibia and fibular nerves were obviously extended (normal range: 42 - $50 \mathrm{~ms}$ );

5) CT scan showed that the transverse and sagittal diameters of the dural sac were $\leq 12 \mathrm{~mm}$ (normal transverse diameter: 13 - $21 \mathrm{~mm}$; normal sagittal diameter: 12 - $16 \mathrm{~mm}$ );

6) MRI scan showed thickened ligamentum flavum and more than 3 intervertebral discs compressing the dural sac. The overall sagittal diameter of the dural sac was shortened by over $3 \mathrm{~mm}$.

Meeting one of the above 1 - 3 and 4, 5, one could incorporate diagnostic criteria.

\section{Methods}

These include curvature adjustment, relaxing the tendons, spinal orthopedic manipulation and functional exercise.

\subsection{Relaxing the Tendons}

Before curvature adjustment, all cases were treated with hot compression of medicine, needling technique (Jiaji Points) and tuina to relax the tendons.

\subsection{Spinal Orthopedic Manipulation and Curvature Adjustment}

\subsubsection{Spinal Orthopedic Manipulation}

After relaxing the tendons, choose rotation of the thoracic and lumbar vertebrae, rotation of the lumbar vertebrae and oblique pulling of the lumbosacral vertebrae of spinal orthopedic manipulation. For specific procedures of these manipulations, please refer to the "Spinal Orthopedics in Chinese Medicine" [3].

\subsubsection{Curvature Adjustment by Four-Direction Traction}

The prone position semi suspension double lower limbs hyperextension thoracic-lumbar vertebrae traction method (Figure 1, Figure 2) by using the four-direction orthopedic device. At the beginning of four-way traction, elevate the lower body to form an angle of $25^{\circ}-45^{\circ}$ with the thoracic-lumbar vertebrae. Then adjust the treatment device to slowly elevate and suspend the limbs. After this, placing the supporting board at approximately $30 \mathrm{~cm}$ away from the limbs and make the lower abdomen leave the board as well (depending on the patient's tolerance), using the thoracic-lumbar joint as the pivot. After traction, ask the patient to stay in bed for same length of time as needed for traction.

Tendon relaxing manipulation and orthopedic curvature adjustment are both conducted once a day. Take one day off after 7 days of treatment. 


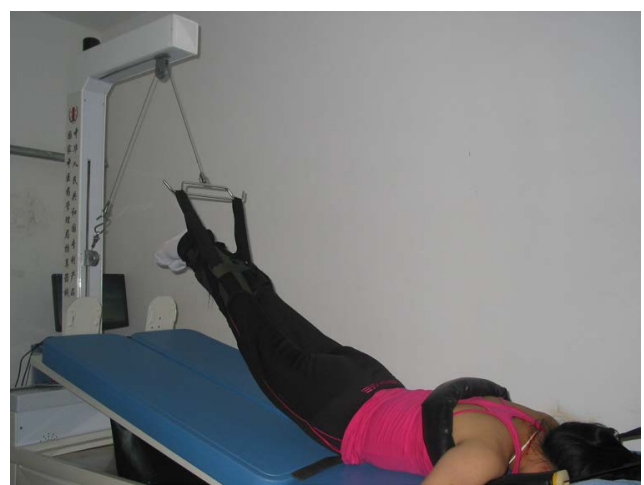

Figure 1. Hyperextension suspension-four-direction curvature adjustment method of traction.

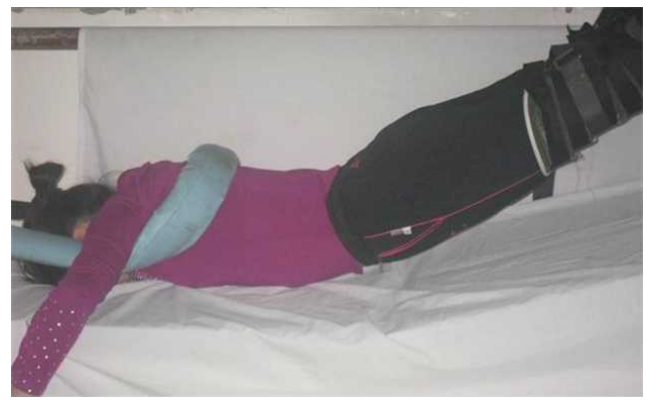

Figure 2. Hyperextension suspension-four-direction curvature adjustment method of traction, the drawing board is placed away from the lower extremities $30 \mathrm{~cm}$, away from pallet appropriate to the abdomen, the fulcrum of power in the thoracolumbar.

\subsection{Functional Exercise}

To sit very belly back hyperextension practice exercises, each time for 20 - 50, two times a day.

\section{Therapeutic Efficacy Observation}

\subsection{Therapeutic Efficacy Evaluation Criteria}

Our research team set up a "100 points evaluation for LSS" according to the therapeutic efficacy criteria, signs, symptoms and imaging quantifications. Using calculated points as indexes, this evaluation contains 5 grades, namely recovery, basic recovery, alleviation, marked effectiveness and failure. Less than 5 points increase after treatment indicates failure; 5 - 10 points increase indicates marked effectiveness; 11 - 20 points increase indicates alleviation; 21 - 30 points increase indicates basic recovery; and 31 - 40 points increase indicates recovery. For cases with a relatively higher baseline, over 80 points in total indicates recovery.

\subsection{0-Point Evaluation Method for LSS}

\subsubsection{Movement of the Lower Limbs (20 Points)}

Grade 0 ( 0 point): Paralysis and inability to stand.

Grade I (5 points): Ability to stand but need support to walk.

Grade II (10 points): Intermittent claudication, i.e., numbness, weakness, soreness, distension and pain of the lower limbs, needs rest after walking less than 100 meters.

Grade III (15 points): Intermittent claudication occurs after walking 100 - 500 meters.

Grade IV (20 points): Absence of obvious pain in the lower limbs and asymptomatic within 500 meters of walking.

\subsubsection{Tactile Sensation Grading (5 Points)}

Grade 0 (0 point): no sensation at all. 
Grade I (1 point): presence of deep touch sensation.

Grade II (2 points): presence of pain sensation and some touch sensation.

Grade III (3 points): complete pain and touch sensations.

Grade IV (4 points): complete pain and touch sensations with an ability to identify different sensations in distant locations.

Grade V (5 points): normal sensation.

\subsubsection{Movement of the Low Back (5 Points)}

0 : loss of lumbar movement or reluctant to move because of pain.

1: restricted lumbar movement with an approximately $60^{\circ}$ of restricted range of motion.

3: restricted lumbar movement with tolerable pain and an approximately $30^{\circ}-60^{\circ}$ of restricted range of motion.

4: slightly restricted lumbar movement with an approximately $10^{\circ}-30^{\circ}$ of restricted range of motion.

5: almost normal lumbar movement.

\subsubsection{Tendon Reflex (10 Points, either Patellar Reflex or Achilles Tendon Reflex)}

0 : absence of reflex or positive sign of straight leg raising test $\left(<45^{\circ}\right)$.

5: decreased reflex or positive sign of straight leg raising test $\left(45^{\circ}-60^{\circ}\right)$.

10: Presence of reflex or negative sign of straight leg raising test.

\subsubsection{Muscle Strength Measurement (10 Points) (Instructions: For Patients That Can Walk,}

Examine Them in the Case of Intermittent Claudication during Movement)

Grade 0 (0 point): Complete muscle paralysis and absence of muscle contraction through observation and palpation.

Grade I (2 points): Voluntary muscle contraction fails to move the joint.

Grade II (3 points): Muscle contraction can move the joint horizontally but fails to counteract the gravity.

Grade III (6 points): Voluntary joint movement during counteracting the gravity but fails to move with resistance.

Grade IV (8 points): Ability to move with major resistance but still weaker than normal.

Grade V (10 points): normal muscle power.

\subsubsection{Bladder Function (10 Points)}

0 : urine retention.

2: extremely difficulty in micturition, incontinence or dripping of urination.

3: difficulty or strain in micturition.

8: frequent and hesitant urination.

10: normal bladder function.

\subsubsection{Radiographic Curvature Scoring (30 Points)}

Grade V (0 point): the arch area: 0 , minus or $>38 \mathrm{~cm}^{2}$; appearance: reversed arch; arched upper part but straightened or extended lower part; lumbosacral shaft angle: $<110^{\circ}$ or $>150^{\circ}$.

Grade IV (5 points): the arch area: 0 ; appearance: straightened; $110^{\circ} \leq$ lumbosacral shaft angle $<120^{\circ}$ or $145^{\circ}$ $<$ lumbosacral shaft angle $\leq 150^{\circ}$.

Grade III (15 points): $0<$ the arch area $\leq 16 \mathrm{~cm}^{2}$; appearance: markedly decreased or arched upper part with flexed lower part; $120^{\circ} \leq$ lumbosacral shaft angle $<125^{\circ}$ or $140^{\circ}<$ lumbosacral shaft angle $\leq 145^{\circ}$.

Grade II (25 points): $16 \mathrm{~cm}^{2}<$ the arch area $<28 \mathrm{~cm}^{2}$; appearance: decreased; $125^{\circ} \leq$ lumbosacral shaft angle $<130^{\circ}$ or $135^{\circ}<$ lumbosacral shaft angle $\leq 140^{\circ}$.

Grade I (30 points): $28 \mathrm{~cm}^{2} \leq$ the arch area $\leq 38 \mathrm{~cm}^{2}$; appearance: normal; $130^{\circ} \leq$ lumbosacral shaft angle $\leq 135^{\circ}$.

\subsubsection{MRI Dural Sac Capacity (10 Points, for the Spinal Canal Pattern) (Instructions: Calculate the} Capacity for Total Compressed Spinal Segments Using T2W1 and Im5 That Are Consistent before and after Treatment)

0: $>3 \mathrm{~mm}$ of the compressed spinal segment before treatment. After treatment, add 5 points for $1 \mathrm{~mm}$ increase. 
The maximum score is 10 points.

\subsubsection{CT Lateral Recess Capacity (10 Points, for the Radicular Canal Pattern)}

0: $<5 \mathrm{~mm}$ (normal range: 5 - $7 \mathrm{~mm}$ ) of the compressed lateral recess capacity before treatment. After treatment, add 5 points for $2 \mathrm{~mm}$ increase. The total score is 10 points (Note: use MRI or CT alternatively).

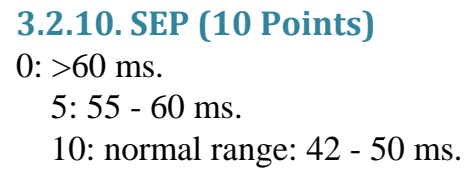

\section{Results and Statistical Method}

The SPSS 13.0 version software was used for statistical analysis on the 100-point LSS and curvature evaluation, and the $t$-test was used for independent samples on a total of 90 cases. The $p<0.05$ indicates a statistical significance.

The 100-point evaluation and curvature grading before and after treatment (Table 1).

Non-parameter test on two independent samples using the statistical software has shown that there were statistical significances in both 100-point evaluation $(t=-26.01, p<0.01)$ and curvature grading $(t=-14.34, p<$ 0.01 ) before and after treatment. In addition, the LSS signs, symptoms and curvature grading were all significantly increased after treatment.

After the four-direction traction for curvature adjustment in 90 LSS cases, the overall response rate was $98.9 \%$, including a clinical recovery rate of $83.3 \%$, response rate of $13.3 \%$, marked effectiveness rate of $2.2 \%$ and failure rate of $1.1 \%$. The spinal curvatures were improved in $81 \%$ of the patients. The 3 -month follow-up showed that the "excellent" and "very good" rates of the health status and signs \& symptoms improvement were $98.8 \%$ and $96.4 \%$ respectively. Clinical trials have proven that patients are willing to accept this safe reliable treatment technique.

\section{Case Study}

A 35-year-old female patient first visited on April 15, 2010 because of "2 years of intermittent low back pain that aggravated and radiated numbness and pain in the left leg for over 4 months”. Her signs and symptoms: low back pain, especially the sensations of soreness and distension, restriction in rolling over the body, and an inability to bend or sit (no more than 5 minutes), stand (no more than 10 minutes) or walk (no more than 300 meters) for long period of time.

Other symptoms include: leg numbness radiating down to the toes, numbness around the anus and perineum, and cold intolerance below the waist. Physical examination showed that lumbar muscle stiffness with poor elasticity, tenderness along the bilateral sides of L4-S1, positive sign of straight leg raising test (left leg: $30^{\circ}$; right leg: $50^{\circ}$ ), decreased patellar tendon reflex on the left side, absence of Achilles tendon reflex on both sides, leftsided weakness by great toe dorsal extension test, grade III muscle strength of the left leg and grade IV of the right leg, decreased muscle tone on both legs, and atrophy of left-sided gluteal and leg muscles. The tongue was pale red with a thin white coating. The pulse was deep, thready and weak.

The lumbar MRI done on January 19, 2010 showed (see Figure 3): L4/5 disc herniated $7 \mathrm{~mm}$ to the back and left, compressing the dural sac. The bilateral intervertebral foramen was narrowed and the longitudinal diameter of the spinal canal was 1mm.The lumbar PA X-ray film done on April 7, 2010 showed (see Figure 4): spinous processes of the lumbar vertebrae deviated to the right side, narrowed intervertebral space of L4-5 and L5-S1, disappeared lumbar curvature and presence of bilateral shadows on the posterior borders of each vertebra.

Diagnosis: lumbar spinal stenosis.

The patient was treated with curvature adjustment using the "Four-direction orthopedic traction bed" (see Figure 1 and Figure 2) coupled with tendon-relaxing manipulation and functional exercise. After 56 times of treatment, her symptoms all disappeared. The lumbar P-A X-ray film showed an absent rotation of the lumbar vertebrae and a normal lumbar curvature (see Figure 5). The lumbar MRI scan showed decreased herniation (back and left) of L4/5 disc, spinal broadening $3 \mathrm{~mm}$ (Figure 6). The 3-month follow-up showed "excellent" rates for health status, signs and symptoms. 
Table 1. The 100-point evaluation and curvature grading of 90 LSS cases before and after treatment $(\bar{x} \pm \mathrm{s})$.

\begin{tabular}{cccc}
\hline Item & Pre-treatment score & Post-treatment score & $p$ value \\
\hline Curvature & $11.61 \pm 7.71$ & $21.28 \pm 6.64$ & 0.00 \\
100-point evaluation & $47.81 \pm 11.52$ & $78.53 \pm 9.06$ & 0.00 \\
\hline
\end{tabular}

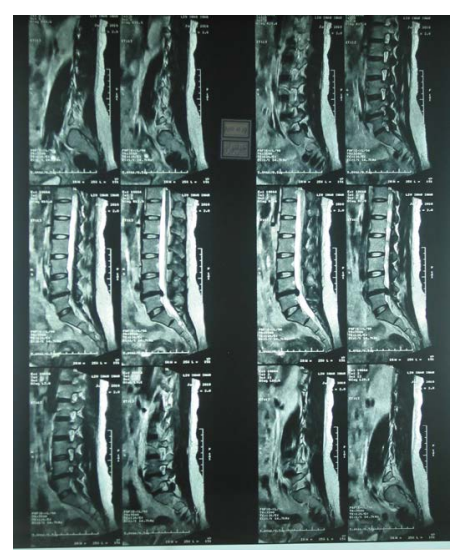

Figure 3. Before treatment: MRI showed that disc herniation of L3, 4, 5 compressing the dural sac, especially L5. The total compressed sagittal surface of the spinal canal was $7 \mathrm{~mm}$.

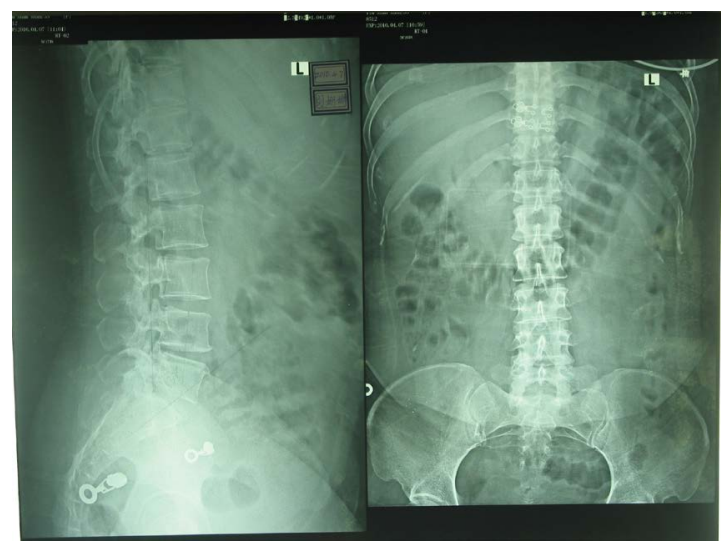

Figure 4. Before treatment: X-ray film P-A view lumbar left bending of $10^{\circ}$; lateral view: rotation of lumbar vertebrae above L4, bilateral signs, grade IV of the spinal curvature.

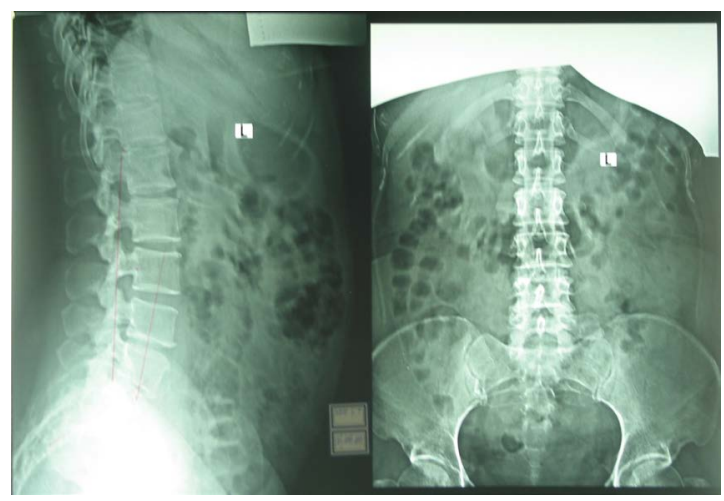

Figure 5. After treatment: Lumbar X-ray film P-A view showed disappeared left bending; Lateral view showed disappeared rotation, grade II of the spinal curvature. 


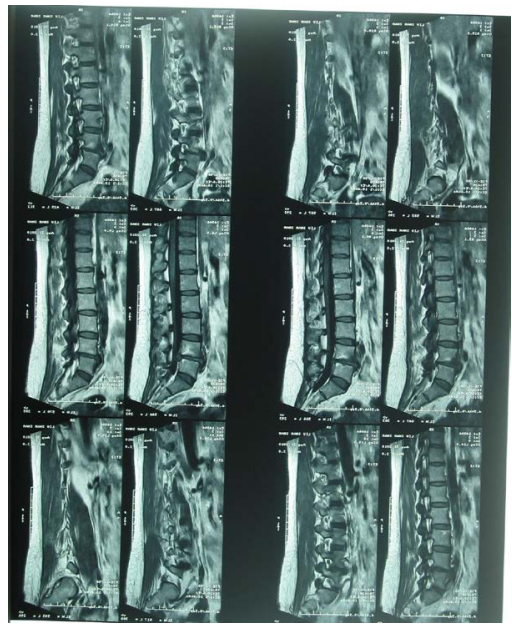

Figure 6. After treatment: Lumbar MRI showed disc herniation of L3, 4, 5 compressing the dural sac. The total compressed sagittal surface of the spinal canal was $4 \mathrm{~mm}$, i.e., increased by $3 \mathrm{~mm}$, especially at L5.

\section{Discussion}

The main pathology of LSS lies in lumbar vertebral articular disorders and subsequent protruding of discs into the spinal canal, and buckling and thickening of the posterior longitudinal ligament and ligamentum flavum. This may further change the spinal curvature and narrow the spinal canal, intervertebral foramen and lateral recess, compressing the spinal cord and nerve root and leading to conduction disorders. Patients may present lower back pain and so on of a series of symptoms and signs. We believe that the human lumbar curvature is gradually formed with development since standing and walking at the age of 1 [4]. Actually, the spinal curvature decides the capacity of the spinal canal and size of the lateral recess; and psoas major muscle serves as the major force for this curvature [5]-[7]. D. F. Kader [8] believed that the multifidus muscles supported the lumbar curvature by conducting the longitudinal pressure to the anterior longitudinal ligament like a bow string. This indicated that the working force of lumbar curvature could be influenced by (low) back muscles at the posterior border, and more by the psoas major muscle at the anterior border.

Through measurement on cross-sectional area of the psoas major muscle in normal volunteers and patients herniated lumbar discs, Dangeria, T.R. et al. [9] found the cross-sectional area of the psoas major muscle in patients with disc herniation. Through MRI scan study in patients with lower back pain, Barker, K.L. et al. [10] found that the decrease in cross-sectional area of the psoas major muscle was associated with persistent symptoms; and that the movement of psoas major muscle was restricted on the affected side (with symptoms).

Most LSS patients experience a smaller, disappeared or even reversed lumbar curvature. This is mainly due to injury, adhesion or atrophy of the psoas major muscle. Higuchi, K. et al. [11] found that the psoas major muscle had two communicating branches: superficial oblique branch and deep transverse branch. The superficial oblique branch connects with the sympathetic nerve trunk and spinal nerves of T12-L2, whereas the deep transverse branch is adjacent to the vertebrae. As a result, patients with LSS often present progressively worsening symptoms over time. The hyper-extension traction in this clinical trial aims to balance the strength of psoas major muscle, restore its isometric contraction, adjust the curvature and thus improve the stenosis of spinal canal and lateral recess.

Curvature-adjustment orthopedic procedure mainly works on adjusting the spinal curvature, in other words, on restoring the biomechanical changes of the spine due to chronic strain-related rotation, tilting and scoliosis of the lumbar vertebral articulations. Functional exercise further helps to strengthen and facilitate the muscles and ligaments. Taken in this sense, exercise is a key supplementary method for curvature adjustment and also an essential measure to prevent relapse of LSS.

\section{Acknowledgements}

We are grateful to China's state administration of traditional Chinese medicine project funding this research 
topic, and Chaozhou Central Hospital affiliated to Southern Medical University and Foshan Hospital of TCM Guangdong Province in observation of clinical cases in this paper.

\section{References}

[1] Herkowitz, H.N., et al. (2004) The Lumbar Spine. 3rd Edition, Lippncott Williams \& Wilkins, Philadelphia, 11-27, 472-489.

[2] Wei, Y.-Z. (2006) Spinal Orthopedics in Chinese Medicine. People’s Medical Publishing House, Beijing, 140-154.

[3] Wei, Y.-Z. (2006) Spinal Orthopedics in Chinese Medicine. People’s Medical Publishing House, Beijing, 142-172.

[4] Wei, Y.-Z., Sun, Y.Z., Wei, C.D., et al. (2005) Dynamic X-Ray Film Investigation of Relationship between the Role of Musculus Psoas Major and Lumbar Curvature. Chinese Journal of Clinical Anatomy, 23, 579-582.

[5] Wei, Y.-Z., Wei, C.D., Wei, Y.F., et al. (2005) The Experimental Study on the Relationship between the Psoas and the Lumbar Curve. Chinese Journal of TCM and Pharmacy, 20, 212-214.

[6] Wei, Y.-Z., Xie, B., Tan, S.S., et al. (2008) The Tension Effects of Psoas Muscle on the Extended Stress of the Spinal Biomechanical Research. Chinese Journal of Clinical Anatomy, 26, 543-546.

[7] Wei, Y.-Z., Tian, X.Y., Wang, H.M., et al. (2011) Experimental Biomechanics Study on the Relationship of Psoas Major Muscles and Lumbar Spine Movements. Chinese Journal of Clinical Anatomy, 29, 97-99.

[8] Kader, D.F. (2000) Correlation between the MRI Changes in the Lumbar Multifidus Muscles and Leg Pain. Clinical Radiology, 55, 145-149. http://dx.doi.org/10.1053/crad.1999.0340

[9] Dangeria, T.R. and Naesh, O. (1998) Changes in Cross-Sectional Area of Psoas Major Muscle in Unilateral Sciatica Caused by Disc Herniation. Spine, 23, 928-931. http://dx.doi.org/10.1097/00007632-199804150-00016

[10] Barker, K.L., Shamley, D.R. and Jackson, D. (2004) Changes in the Cross-Sectional Area of Multifidus and Psoas in Patients with Unilateral Back Pain: The Relationship to Pain and Disability. Spine, 29, E515-E519. http://dx.doi.org/10.1097/01.brs.0000144405.11661.eb

[11] Higuchi, K. and Sato, T. (2002) Anatomical Study of Lumbar Spine Innervations. Folia Morphologica, 6, 71-79. 